\title{
Isolation and Identification of Streptococcus Suis
}

\author{
Jinxiu Mu \\ Weifang University of Science \& Technology, Shandong, Shouguang, 262700, China.
}

Keywords: Streptococcus suis serotype 2; Identification; Virulence; Drug sensitivity test.

\begin{abstract}
This study mainly isolated and identified a Streptococcus suis strains by the methods of disease material separation, microscopic examination and PCR identification, drug sensitivity test, biochemical test, animal attack to xicity test and so on. Get A preliminary assessment of its virulence and drug resistance to provide a reference basis for further study of Streptococcus suis.
\end{abstract}

\section{Introduction}

Streptococcus suis is a major disease causing harm to the pig industry. The pathogen is detected by the nose fluid, urine, blood, muscle, visceral and joint flu id of the pig. The death of pork, viscera and waste without the harmless treatment are the important source of the disease. Respiratory tract is the major route of transmission. The disease can occur in the four seasons, but the incidence of 4-10 months, often endemic, the short term can spread to the entire group, the incidence, mortality is high. This experiment is mainly from pig disease viscera disease data collection of strains, selection and validation of Streptococcus suis, test the resistance and pathogenic of further prevention and treatment of swine diseases and deep research provide basic information and materials.

\section{Materials and methods}

Isolation and microscopic examination of pathogen. The brain, lung and liver disease collect dead pigs inoculated in the normal tissues of sheep blood agar plate. After bacterial staining, microscopic examination, purification, 1 strains of suspected strains, named DYL-22. And hemolysis test is conducted. Among them, there are gram stain, staining, microscopic examination, purification steps are: smear, drying, fixation, staining, microscopic examination, purification.

Sugar fermentation experiment. The experimental strains access glucose, maltose, fructose, lactose, beta galactosidase (ONPC), inulin, arabinose, thamnose, inositol, Dulcitol, mannitol, sorbitol, xylose biochemical reagent tube sealing $37^{\circ} \mathrm{C} 24 \mathrm{~h}$ and observe the result of the experiment.

Drug sensitivity test. According to standard paper agar diffusion (K-B) operation, the drug sensitive paper is attached to the blood of the pathogenic bacteria on the blood plate, keep the appropriate distance between the paper, 37 temperature boxes 24 hours. The experiment shared 9 drugs: AN, GM, CRO, AMS, N, LVF, SMX, FOS, F.

Identification of pathogenic bacteria PCR. The content of PCR identification in this experiment primarily aims at the test whether the strain DYL-22 contained GDH gene. Streptococcus suis glutamate dehydrogenase belongs to GDH protein family, which raises on the cell surface. Nucleic acid sequence analysis shows that GDH genes includes an open reading frame, encoding 448 amino acid residues and expressed on the cell surface. The enzy me activity is dependent NAD $(\mathrm{P}) \mathrm{h}$, substrate for L-glutamic acid, its coding gene is SS2 (type 2 Streptococcus suis) conserved. SS2 GDH has conserved antigenic and infection of virulent Streptococcus suis in pigs serum reaction, detection of Streptococcus suis as an important indicator of the antigen. If the GDH gene is achieved, then the strain of Streptococcus suis is possibe for Streptococcus suis type 2. Synthesis of Streptococcus suis type 2 specific primers, respectively gdh688a: CCATGGACAGATAAAGATGG, gdh689b: GCAGCGTATTCTGTCAAACG.

PCR system, condition and detection 
PCR reaction system (20ul system) is: Taq 10ul Premix, template 1ul, upstream and downstream primer 1ul,Ultra pure water $7 \mathrm{ul}$, a total of $20 \mathrm{ul}$.

The reaction conditions of PCR were: at $94^{\circ} \mathrm{C}$, the den aturation of $5 \mathrm{~min}$, then entered in to 35 cycles: $94^{\circ} \mathrm{C} 1 \mathrm{~min}, 51^{\circ} \mathrm{C} 1 \mathrm{~min}, 72^{\circ} \mathrm{C} 1 \mathrm{~min}$, at last 7 min extends at $72^{\circ} \mathrm{C}$, and the product of PCR is kept at $4^{\circ} \mathrm{C}$ for a brief. Agarose gel electrophores is detection.

Pathogenic bacteria virulence experiment. Blood agar inoculum activation is picked from a single colony from Strep tococcus vaccination in Ma Dingtang (plus $4 \%$ heme) and $37^{\circ} \mathrm{C}$ culture $16-18$ $\mathrm{h}$, viable bacterial counts. Take soup Martin medium $0.2 \mathrm{ml}$ (including bacteria about 40 million) intravenous injection of $1.5 \sim 2.0 \mathrm{~kg}$ rabbits, two rabbits are immunized. At the same time, live in bacteria count; blank control group is set to 2 and injected with saline. After the death of the rabbit, rabbit an atomy, and take blood inoculation blood incline $37^{\circ} \mathrm{C}$, cultured for $24 \mathrm{~h}$. At the same time, the rabbit blood filling small tube, $-20^{\circ} \mathrm{C}$ preservation.

\section{Results and analysis}

The results and analysis of the mirror. The diseased inoculates in the blood culture medium, the bacteria separation and purification, observe suspicious strains grew well, colonies are round, smooth transparent ridges, grey dew like small colonies and produce obvious beta hemolysis (Fig. 1). Suspicious colonies on the blood culture base are inoculated on the agar medium of wheat Kang Kai ag ar and has no bacterial growth. The growth of colonies on a common medium is poor. Picking blood culture built on the typical colonies, Gram staining, microscopic examination, visible gram positive, short chain sor in single coccu s cell diameter 0.1-0.2um (Fig. 2 ).

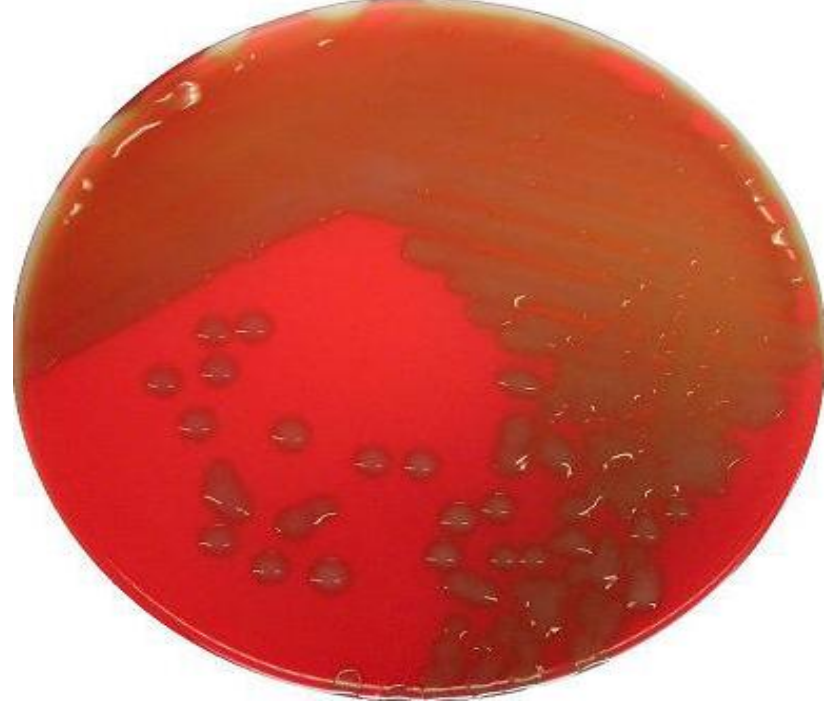

Fig. 1 hemolysis test 


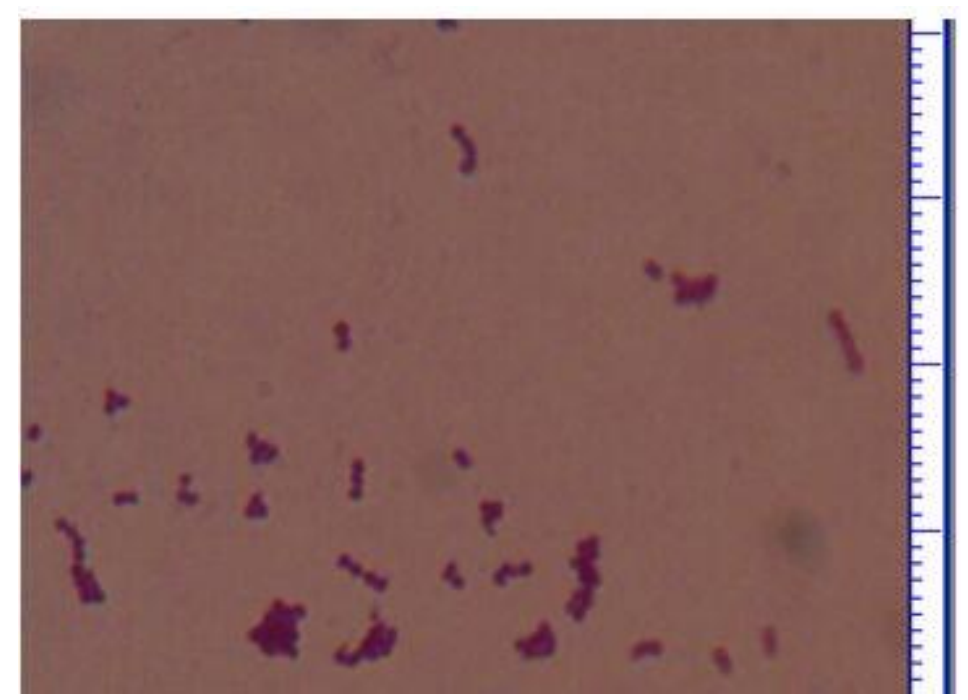

Fig. 2 gram stain amplification factor of 1000

Biochemical test results and analysis. Mainly for the sugar fermentation of Streptococcus suis biochemical tests, from Fig. 3, Fig. 4 and Fig. 5, it can be seen that biochemical test of glucose, fructose, maltose, lactose pipe by blue and purple tum yellow, inulin from light pink and the color of biochemical test tube are not found in the gas. Thus, the pathogen can be fermented more than five carbohydrates, but acid production but not gas.

The ONPC, arabinose, rhamnose, inositol, Dulcitol, mannitol, sorbitol, xylose biochemical test tube did not change also do not produce gas, indicating that the pathogenic bacteria cannot glycolysis above carbohydrates.

The results of the experiment are basically the same as the expected results of the experiment.

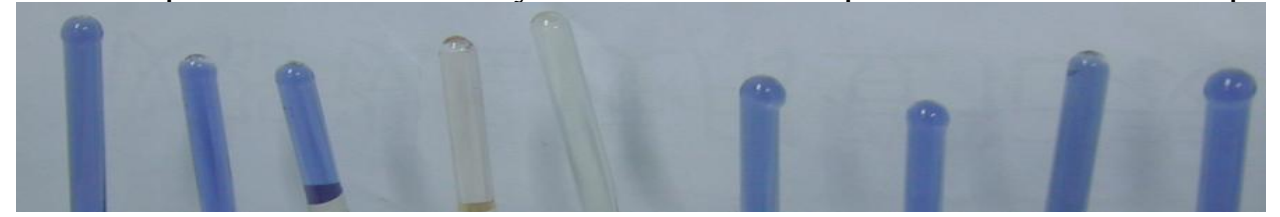

Fig. 3 Pathogen inoculation $0 \mathrm{~h}$

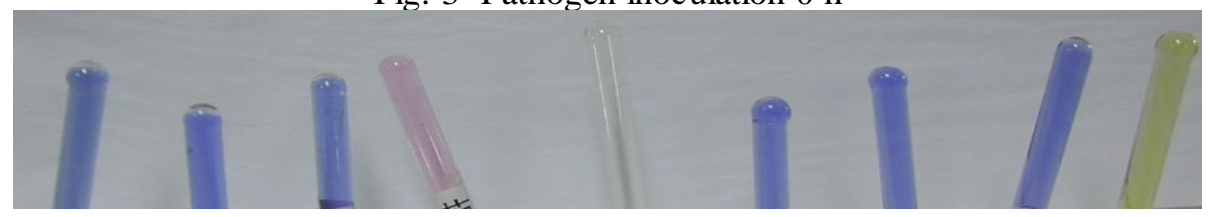

Fig. 4 Pathogen inoculation $24 \mathrm{~h}$

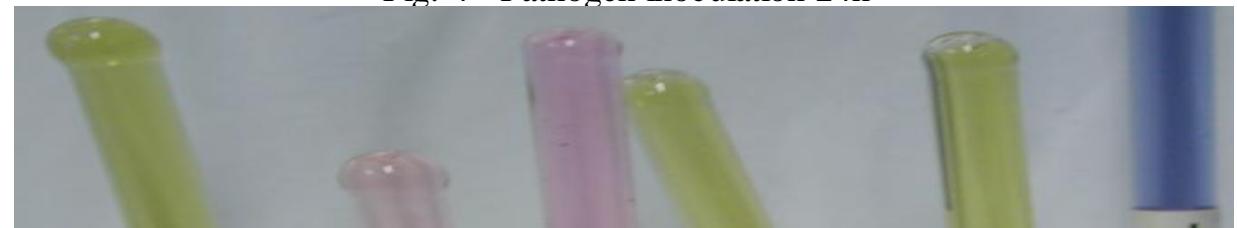

Fig. 5 Pathogen inoculation $24 \mathrm{~h}$

Drug sensitivity test results and analysis. Fig. $6:$ drug sensitive paper from the clockwise direction are CRO, AN, N, FOS, AMX, F, LVF, SMX. In the middle is: GM.

The neomycin $(\mathrm{N})$, amikacin $(\mathrm{AN})$, gentamicin (GM), sulfamethoxazole (SMX) of the pathogen without or inhibitory effect is weak and other antibacterial drugs ring diameter respectively is: CRO:3.040cm, F:2.460cm, FOS:2.360cm, AMX:1.610cm, LVF:1.734cm.

The pathogen of neomy cin, amikacin, gentamicin, sulfamethoxazole sensitive deg ree is low, highly resistant, high sensitivity to ceftriaxone, fluorescein test, tobramycin, amoxicillin and levitation, resistance is weak, the clinical medication can be for reference. 


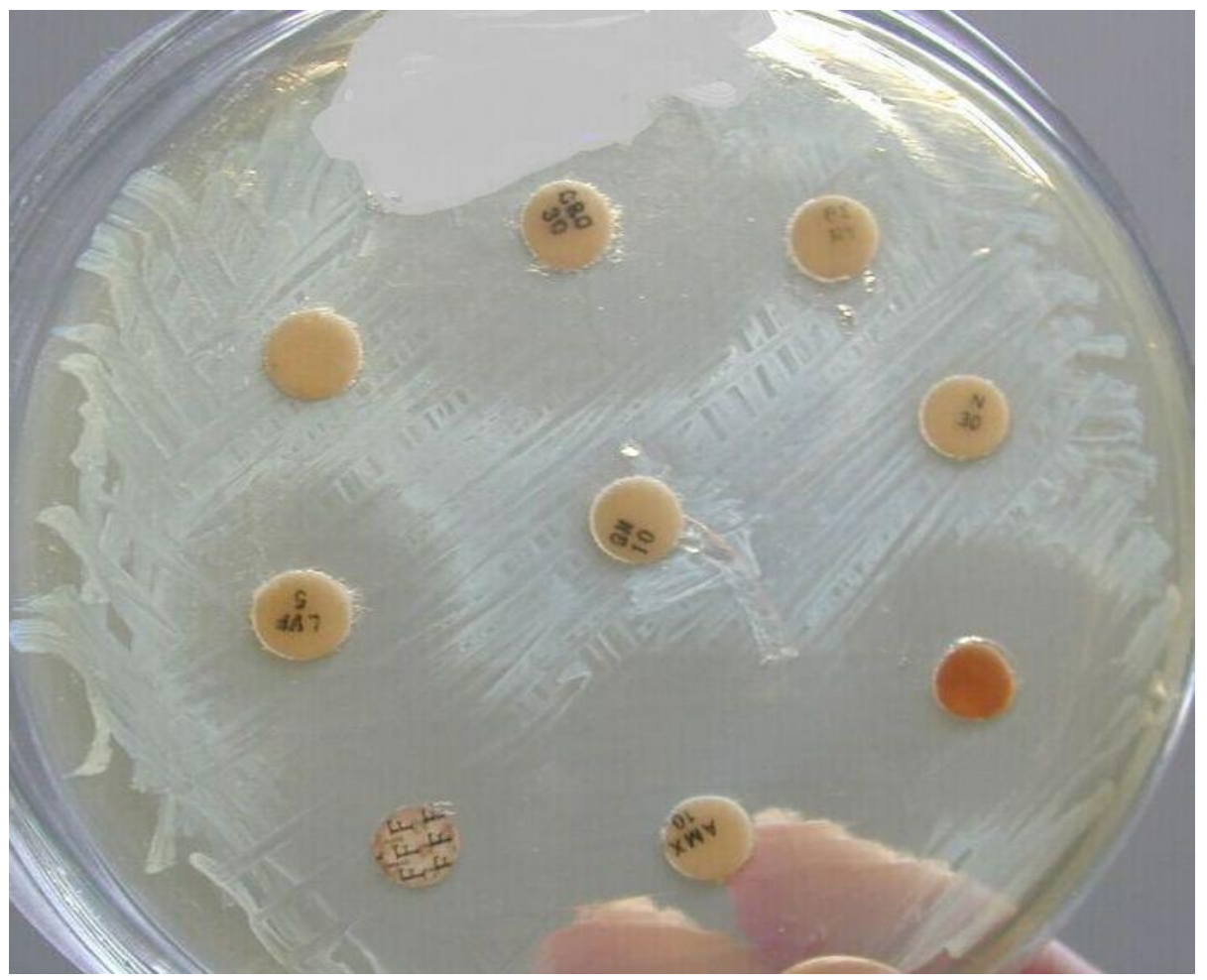

Fig. 6 Pathogenic bacteria drug sensitivity test

PCR electrophoresis results and analysis. PCR products are segregated on agarose gel electrophoresis. The results show that the standard of Streptococcus suis and isolates DYL-22 are about 700bp appear to strip, consistent with the expected frag ment.

The results and analysis of virulence experiments. The symptoms of respiratory distress were around $36 \mathrm{~h}$, and the death of $72 \mathrm{~h}$ was around. Necropsy: typical pneumonia, meningitis, myocardial hemorrhage, mesenteric hemorrhage, necrosis of spleen enlargement, renal hemorrhage and necrosis; blank control, observe two weeks Houjian live.

\section{Conclusions}

Streptococcus suis usually in pig tonsils settlement can be divided into 35 serotypes. In pathog en ic serovars, type 2 is the most virulent, incidence of a disease distinguished, wide range of popular, followed by type 1 and type 9 .

Microscopic examination of colony morphology and hemolysis and Streptococcus compliance; biochemical test results, in addition to the ONPC and inulin and other reagent tube in line with expectations; by PCR detection of the pathogen indeed with gdh gene, gdh gene characteristic of Streptococcus suis genes. Therefore, pathogenic bacteria for Streptococcus suis.

The animal virus attacking test and rabbit organs show obviously Streptococcus suis type 2 infection symp toms, lung hemorrh age, mesenteric lymph node hemorrhage, enlarg ement of the spleen, brain hemorrhage and edema, cardiac and renal lesions.

\section{References}

[1] Staats J J,Feder I, Okwumabua O,et al.Streptococcus suis past and present [J]. Vet Res Commu m, 1997, 21:381-407.

[2] Gott schalk M, Petibois S, Higgifls R, et al. Adherence of Streptococcus suis cap sular type 2 to porcine lung sections[J]. Can J Vet Res, 1991,55(3):302-304.

[3] Yan xiu Ni, Jichun Wang, Kongwang He. [J]. molecular epidemiology of Streptococcus suis zoonosis in China Journal, 2002, 18 (5):45-47. 
[4] Williams A E. Relationship between intracellular survival in macrophages and pathogenicity of Streptococcus suis type 2 isolates[J]. Microb Pathogenesis, 1990, 8(3): 189-196.

[5] Zhitao Duan, Kongwang He, Yan xiu Ni. Four strains of Streptococcus suis type 2 is olated strains of major virulence factors (MRP, EF) gene sequence analysis [J]. Jiangsu Journal of Agricultural Sciences, 2007,23 (3): 207-209.

[6] Smith HE, Vecht U, Wisselink HJ, et al. Mutants of Streptococcus suis types 1 and 2 impaired in expression of muramidase-released protein and extracellular protein induce disease in newborn germfree pigs. Infect Immun, 1996, 64 (10) : 4409-4412.

[7] $\mathrm{Yu} \mathrm{Ou,} \mathrm{Chengping} \mathrm{Lu.} \mathrm{Streptococcus} \mathrm{suis} \mathrm{type} 2$ vinulence associated protein [D]. Microbial Journal.2002, 22 (2):203 - 206.

[8] Fabisiak M, Kita J, Jedrvczko R,et a1. Prevalence of the Suilysin Gene in Streptococcus suis Strains Isolated fromDiseased and Healthy Carrier Pigs[J]. Pol J VetSci, 2005, 8(2):141-145.

[9] Lil Duan i, Chi Xiao, Sanjie Cao. Progress in virulence factor of Streptococcus type 2 Streptococcus [J]. China animal and animal co morbidity, 23, 2007 (1): 89-91.

[10] Matsuo H,Sakamoto S.Purulent meningitis caused by Streptococcus suis in a pig breeder [J]. Kansenshogaku Zasshi, 2003,77(5):340-342. 